\title{
Influence of stimulus symmetry on visual scanning patterns*
}

\author{
PAUL J. LOCHER $\dagger$ and CALVIN F. NODINE \\ Temple University, Philadelphia, Pennsylvania 19122
}

\begin{abstract}
Eye movements of four Ss performing a complexity rating task in which the stimuli consisted of random shapes differing in symmetry, number of turns (sides) in the perimeter, and structural angularity were examined. It was found that for both symmetrical and asymmetrical shapes, the number of fixations and fixation time increased directly with structural complexity (number of sides). Distributions of fixations for symmetrical shapes clustered in one-half of the shapes, while the distributions of fixations for asymmetrical shapes did not exhibit this one-sided bias. No differences were found in the distributions of fixation time to either half of asymmetrical or symmetrical shapes. The findings suggest that $S$ utilizes an organizing code in addition to the featural code in characterizing a given shape. The organizing code permits $\mathbf{S}$ to generate the feature code for a given shape on the basis of partial information.
\end{abstract}

Studies of the psychophysics of form have repeatedly shown that the symmetry of a pattern is a major form parameter. A variety of studies have been performed to examine the relation between stimulus symmetry and stimulus complexity as determinants of informational content of shapes. Various approaches taken to study this question and the results obtained have been reviewed by Michels and Zusne (1965) and Berlyne (1971). The studies cited in these reviews indicate that the informational content of a pattern is decreased by similarities or other interdependences (e.g., redundancies) among structural elements of patterns (e.g., angles, lines, areas). Symmetry is one type of redundancy.

In one of the first studies which systematically examined the role of symmetry as a determinant of stimulus complexity, Attneave (1957) found that symmetrical shapes were judged less complex than asymmetrical shapes with the same total number of turns (sides) by approximately one standard deviation unit. In a more recent study, Day (1968) found that a symmetrical polygon was generally rated by Ss as less complex than an asymmetrical polygon with the same number of sides.

Most of the information about the nature of symmetry as a form parameter has come from studies of subjective scaling of complexity of polygons. Few attempts have been made to examine the question of how redundancy in a visual stimulus influences other forms of behavior. For example, are symmetrical and asymmetrical shapes viewed in the same way or differently?

Zusne and Michels (1962) found that the degree of symmetry of a polygon was not related in any way to the eye-movement scanning patterns of Ss viewing the

\footnotetext{
*The analysis of the filmed eye-movement records was made possible by the generous support of Drs. Harold L. Kundel and George H. Steward, Temple University Medical School.

$\nmid$ Now at Montclair State College, Department of Psychology, Upper Montclair, New Jersey 07043.
}

shapes. On the other hand, Thomas (1963) noted that the search patterns of Ss viewing Rorschach ink blots exhibited some preferences for one side or the other of a symmetrical display. Noton and Stark (1971) have also reported that several of their Ss exhibited a one-sided visual scanning strategy for certain symmetrical shapes used as stimuli.

To date, few attempts have been made to examine systematically the relationship between the configurational property of symmetry of shapes and the visual fixation patterns associated with the viewing of these shapes. If, as Attneave and others have suggested, symmetrical shapes, because of redundant features, contain less information content than do asymmetrical counterparts equated for complexity (here defined as number of sides), then viewing of the former should require fewer fixations and less fixation time than should viewing of the latter. The present study tests this hypothesis.

\section{METHOD}

\section{Subjects}

Two male and two female undergraduate students enrolled in an introductory psychology course served as Ss. Each $\mathrm{S}$ volunteered to participate in the study.

\section{Stimulus Materials}

The stimulus shapes consisted of a series of 24 black-on-white solid polygons constructed using Method 4 of Attneave and Arnoult (1956). These shapes are shown in Fig. 1. The shapes differed on the following form parameters: symmetry (asymmetrical vs symmetrical) and the number of turns (sides) in the perimeter of the form $(4,6,8$, or 12 sides). One set of 8 shapes was constructed of straight lines, another 8 shapes were constructed by replacing all of the angles by arcs, and another 8 shapes were constructed by replacing one-half of the angles with arcs. The position and size of the arcs were determined randomly. Thus, a series of 24 shapes differing in symmetry, number of turns in the perimeter, and structural angularity was used. The size of all shapes was equated, and shapes were reproduced on $35-\mathrm{mm}$ slides. 
1

2

A

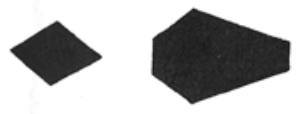

B
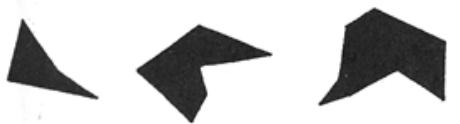

C
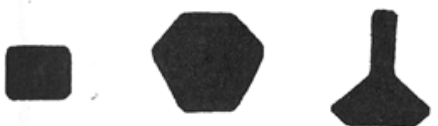

D
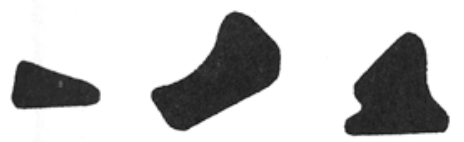

E
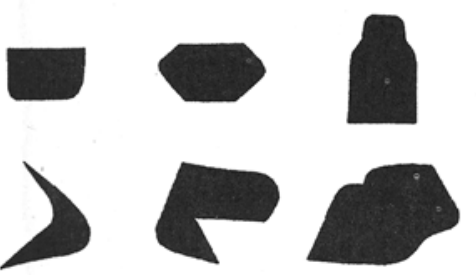

3
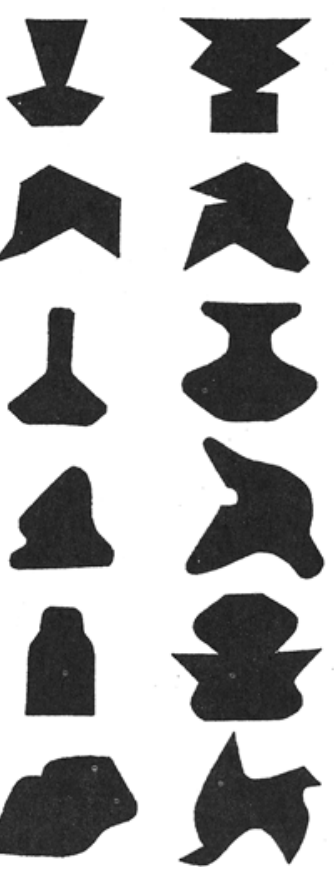

Fig. 1. Twenty-four nonrepresentational shapes used in the experiment. Columns 1, 2, 3, and 4 consist of, respectively, 4-, 6-, 8-, and 12-sided shapes. Row A consists of symmetrical shapes constructed of straight lines. Row B consists of asymmetrical shapes constructed of straight lines. Row $C$ consists of symmetrical shapes constructed of arcs. Row D consists of asymmetrical shapes constructed of arcs. Row $E$ consists of symmetrical shapes constructed of lines and arcs. Row $F$ consists of asymmetrical shapes constructed of lines and arcs.

\section{Apparatus}

The stimuli were presented to Ss via a frosted rear-projection screen using a Kodak Carousel projector positioned $121.92 \mathrm{~cm}$ from the screen. The rear-projection screen was attached to a Polymetric-Eye-Movement recorder, wide angle (Model V-1166). This apparatus was equipped with a $16-\mathrm{mm}$ Beaulieu R-16 reflex motion-picture camera with a Soligor $75-\mathrm{mm}$ f 1.9 Telephoto lens attached to a Paillard-Bolex 40-mm extension tube placed in the viewing port of the recorder housing. Also attached to the recorder housing was a combination chin- and headrest to help hold S's head in position as he examined the stimulus display.

The system described above provided the necessary resolution required to detect multiple fixations on each shape individually projected onto the screen. At a viewing distance of $35.56 \mathrm{~cm}$, the registration accuracy of the position of a visual fixation on the stimulus display was within $2.54 \mathrm{~cm}$ or \pm 2 visual deg. A stimulus shape individually projected upon the screen subtended approximately $36 \mathrm{deg}$ horizontally $(22.86 \mathrm{~cm})$ and approximately $24 \mathrm{deg}$ vertically $(15.24 \mathrm{~cm})$. Thus, it was possible with the set of stimuli to obtain as many as 54 distinct fixations per shape.

The stimulus display, reflected off the right eye of $S$ as he examined the display, was photographed at 8 frames/sec. Figure 2 consists of a section of filmed record showing the stimulus display reflected on the cornea of $S$ as he viewed the display.

The locus of S's gaze was determined by projecting the filmed records onto the surface of a Thomson Table on line with a

LINC-8 computer and locating the $x-y$ coordinates of the fixation relative to the stimulus shape. Details of the data reduction procedure are presented in Kundel and Wright (1969). Using this procedure to analyze the records, it was possible to relate the parameters of visual fixations (number of fixations, duration of fixations, and sequence of fixations) to form details of each of the stimuli, thus enabling $E$ to make inferences about the examination of form features performed by $S$ in completing the experimental task.

\section{Procedure}

The task was described to $S$ as a complexity rating task to engage him in an adequate visual examination of the shape. The $\mathrm{S}$ was instructed to close his eyes as soon as he had decided upon a complexity rating for each shape. This procedure has been found to be an efficient technique for marking the end of a

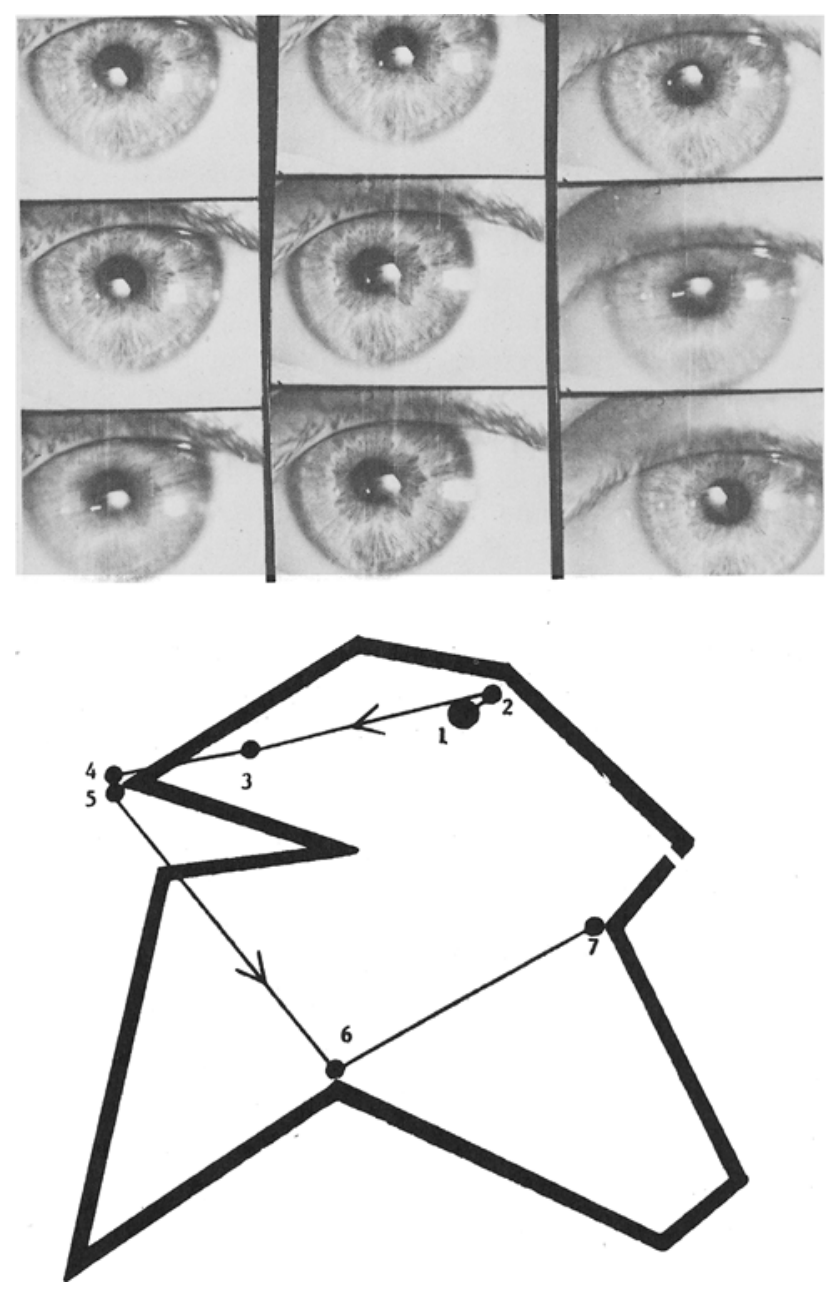

Fig. 2. Section of filmed eye-movement record showing the stimulus display reflected on S's cornea. Starting at the upper left, Frames 1 and 2 show fixations on top center of shape; Frame 3 shows left movement; Frame 4 shows fixation on top left of shape; Frames 5 and 6 show fixations on top left point; Frame 7 shows fixation on lower center indentation of shape; Frame 8 shows right movement; Frame 9 shows fixation on right center indentation of shape. This fixation pattern is illustrated graphically in the lower half of the figure. 
scanning pattern for each shape on the filmed record (Nodine \& Lang, 1971). The $S$ was asked for his complexity rating for each shape before the next stimulus was presented, and these ratings were recorded by $\mathrm{E}$.

The shapes were presented in three groups of eight stimuli, each group being arranged so that it contained all levels of symmetry and number of turns in the perimeter. The distribution of the three levels of structural angularity was randomly distributed among the three groups. The stimuli in each group were the same for each $S$, but the order of presentation and the directional orientation of each of the shapes in each group were randomly determined. The presentation of the first two groups of eight stimuli functioned to familiarize $S$ with the task and the apparatus. Filmed records were made during the presentation of the third group of stimuli. Approximately $10 \mathrm{~min}$ were required by each $S$ to examine all of the shapes of a given group of stimuli. A 5-min "rest" period was used between groups of stimuli.

\section{RESULTS}

Analyses of both quantitative measures (e.g., number of fixations and fixation time) and qualitative measures (e.g., pattern of fixations over stimuli) were carried out.

\section{Quantitative Results}

Each of the dependent variables, number of fixations and fixation time (frames), was tested by analysis of variance using a two-factor repeated-measurements design (Myers, 1972) with two levels of symmetry (asymmetrical and symmetrical) and four levels of shape complexity $(4,6,8$, and 12 sides). The pattern of results was essentially the same for both dependent measures. No differences were found between symmetrical and asymmetrical shapes for either fixations $[F(3,9)=.01$, n.s.] or fixation time (frames) $[F(3,9)=.01$, n.s. $]$. Mean number of fixations and frames for symmetrical and

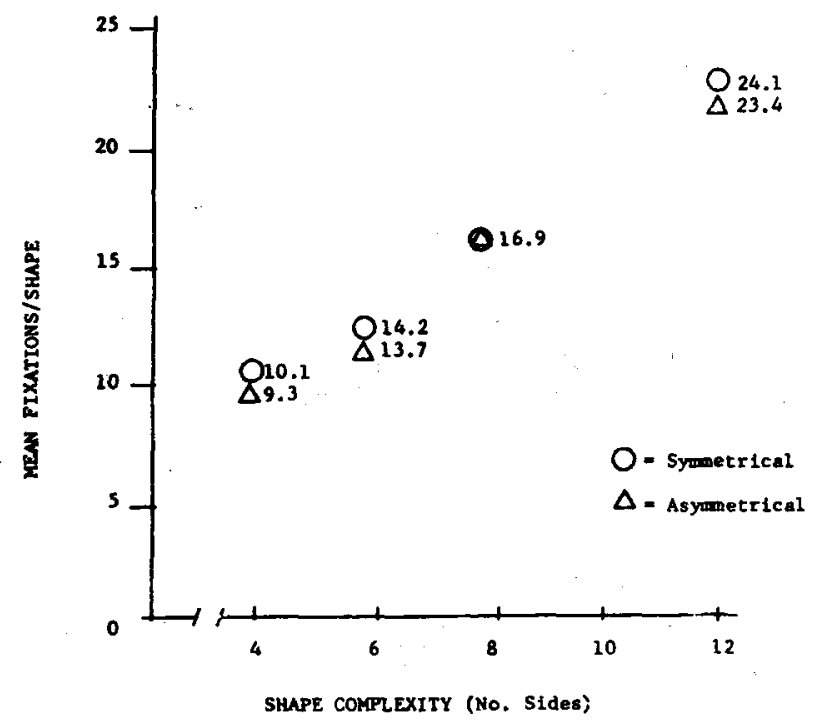

Fig. 3. Mean number of fixations per symmetrical and asymmetrical shape complexity.

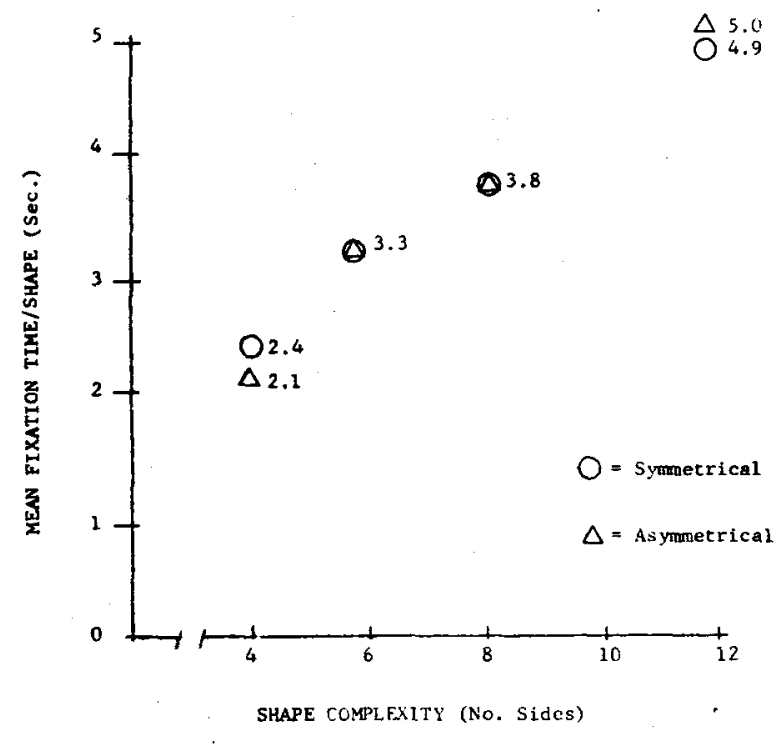

Fig. 4. Mean fixation time (seconds) per symmetrical and asymmetrical shape as a function of shape complexity.

asymmetrical shapes can be found in Figs. 3 and 4. Both fixations and fixation time (frames) increased directly with the levels of shape complexity. For fixations, $F(3,9)=37.97, p<.01$; for fixation time (frames), $F(3,9)=32.98, p<.01$. Figure 3 shows the relationship between shape complexity and fixations, and Fig. 4 shows the relationship between shape complexity and fixation time (frames).

\section{Qualitative Results}

Although there were no differences due to symmetry in either number of fixations or fixation time, there was a difference in the distribution of fixations between symmetrical and asymmetrical shapes. Symmetrical shapes were bisected along the axis of symmetry. Asymmetrical shapes were bisected along the horizontal axis of the shape as viewed by each $\mathrm{S}$. Viewing orientation for both symmetrical and asymmetrical shapes was randomly determined. The distribution of fixations was divided approximately evenly (50-50 and 60-40) between top and bottom halves in 14 out of 16 cases for asymmetrical shapes, but only 5 out of 16 for symmetrical shapes. In contrast, the distribution of fixations for 11 of the 18 symmetrical shapes showed a greater bias (70-30 and above) for one half of the shape relative to the other half.

Despite the fact that the distribution of fixations clustered in one half of symmetrical shapes, it is interesting to note that the distributions of fixation time between halves of symmetrical shapes did not differ from those of asymmetrical shapes $\left(\chi^{2}=3.62, \mathrm{df}=2\right.$, n.s.).

These findings have since been replicated by Locher 
Table 1

Frequency of Biased Distributions of Fixations About the Horizontal Axis for Symmetrical and Asymmetrical Shapes

\begin{tabular}{cccc}
\hline Shapes & $\begin{array}{c}\text { Slight } \\
\text { Bias* }\end{array}$ & $\begin{array}{c}\text { Medium } \\
\text { Bias }\end{array}$ & $\begin{array}{c}\text { Strong } \\
\text { Bias }\end{array}$ \\
\hline Symmetrical & 5 & 8 & 3 \\
Asymmetrical & 14 & 1 & 1 \\
\hline$x^{2}=10.71$ & $d f=2$ & $p<.01$
\end{tabular}

*Slight bias includes $50-50$ and $60-40$ distributions; medium bias, 70-30 and 80-20; and strong bias, 90-10 and 100-0.

(1973) in a study designed to examine the influence of structural complexity on learning and recognition of similar shapes. Locher reported one-sided distributions of fixations and fixation time about the horizontal axes (top-bottom) of symmetrical shapes. No such biased distributions were found for asymmetrical shapes.

\section{DISCUSSION}

Within an information-processing framework, any system for scaling shapes, whether formal (e.g., Leeuwenberg, 1968: Vitz \& Todd, 1971) or intuitive (e.g., Attneave, 1954), includes symmetry as a determinant of structural complexity. The present study provides empirical evidence, in the form of visual fixation patterns, which clarifies the role of symmetry in the scaling of stimulus complexity.

First, both visual fixations and fixation time increased directly with stimulus complexity as measured by the number of sides of the shape. Although the amount of increase in number of fixations and fixation time was nonsignificant among 4,6 , and 8 sides, there was a significant increase in both measures from 8 sides to 12 sides $(p<.05$, Tukey, HSD). This was the case whether the shapes were symmetrical or asymmetrical.

Second, although there were no differences in either number of fixations or fixation time between symmetrical and asymmetrical shapes of comparable information content, the scanning patterns of Ss tended to be one-sided for symmetrical shapes, whereas two-sided scanning patterns characterized asymmetrical shapes. Figure 4 shows some representative visual-fixation patterns for symmetrical and asymmetrical shapes differing in complexity levels. Comparisons of Figs. 5A and 5B (symmetrical shapes) with Figs. 5C and 5D (asymmetrical shapes) provide evidence for the one-sided vs two-sided scanning patterns. In the case of both symmetrical shapes, more attention is shown by Ss on the left half of the shape than on the right half of the shape. In Fig. 5A, almost the entire left-hand perimeter is fixated, whereas only the top of the right-hand perimeter is fixated. The one-sided scanning pattern is even more pronounced in Fig. 5B, where the right-hand perimeter receives no fixations in contrast to the rather detailed scanning pattern over the left-hand perimeter. In contrast, both asymmetrical shapes (Figs. 5C and 5D) show extensive scanning patterns which follow closely almost the entire perimeter of the shape.

Also indicated among the comparisons is the tendency for Ss to fixate on the angle or curved juncture points (or structural features) where two sides meet. The fact that these features receive attention indicates that they

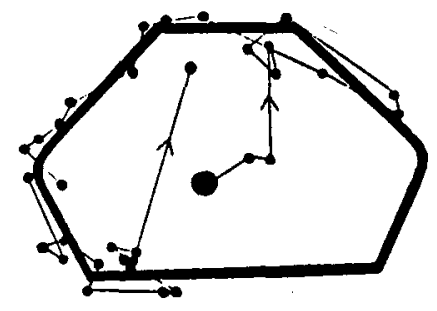

A

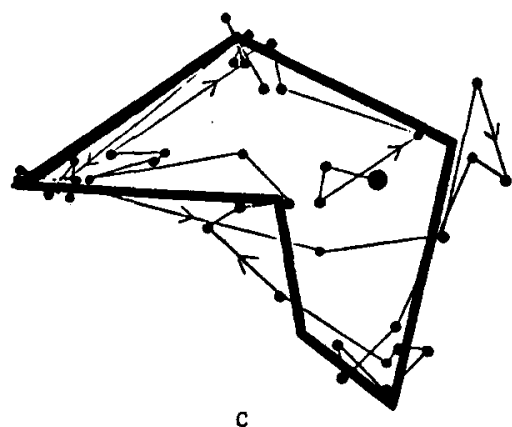

C

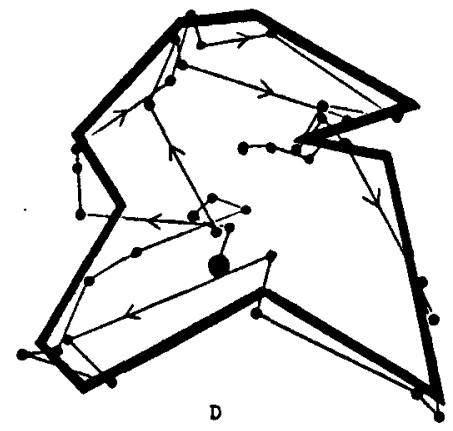

Fig. 5. Representative eye-movement records for symmetrical and asymmetrical shapes differing in complexity. A shows scanning pattern of $\mathrm{SN}$ for 6-sided symmetrical shape. B shows scanning pattern of $S Z$ for 12-sided symmetrical shape. $C$ shows scanning pattern of S D for 8 -sided asymmetrical shape. D shows scanning pattern of $\mathrm{SW}$ for 12-sided asymmetrical shape. 
play a significant role in defining the information content of the shape.

The tendency for Ss to attend to only one half of symmetrical shapes is an interesting finding when viewed within the framework of information theory. Assume each fixation provides $S$ with a featural code for a given shape and an organization code (Simon, 1972). Since the perimeter features in one half of a symmetrical shape are redundant with those in the other half, there would be little point in S's fixating both sides of a symmetrical shape provided $S$ detected, on the basis of partial sampling of featural elements, an organizational principle (code) that would enable him to generate and complete the featural code for that shape. How S detects the organizing code is an interesting question. One obvious answer is that, through peripheral viewing, $S$ picks up information about redundant features of the shape. In the present task, this would mean that $S$ detected redundancies (or lack thereof) without having fixated them. In many cases, the redundant features were $10 \mathrm{deg}$ or more from the point of fixation. Several recent studies from our laboratory (Locher, 1973; Nodine \& Steuerle, 1971; Nodine \& Simmons, 1972) confirm the importance of peripheral viewing during the processing of visual information.

\section{REFERENCES}

Attneave, F. Some informational aspects of visual perception. Psychological Review, 1954, 61, 183-193.

Attneave, F. Physical determinants of the judged complexity of shapes. Journal of Experimental Psychology, 1957, 53, 221-227.

Attneave, F., \& Arnoult, M. D. The quantitative study of shape and pattern perception. Psychological Bulletin, 1956, 53, $452-471$.

Berlyne, D. E. Aesthetics and psychobiology. New York:
Appleton-Century-Crofts, 1971.

Day, H. The importance of symmetry and complexity in the evaluation of complexity, interest, and pleasingness. Psychonomic Science, 1968, 10, 339-340.

Kundel, H. L., \& Wright, D. J.The influence of prior knowledge on visual search strategies during the viewing of chest radiographs. Radiology, 1969, 33, 315-320.

Leeuwenberg, E. L. J. Structural information of visual patterns. The Hague: Mouton, 1968.

Locher, P. J. Influence of structural complexity on eye movement patterns during learning and recognition of two-dimensional shapes. Unpublished doctoral dissertation, Temple University, 1973.

Michels, K. M., \& Zusne, L. Metrics of visual form. Psychological Bulletin, 1965, 63, 74-86.

Myers, J. L. Fundamentals of experimental design Boston: Allyn \& Bacon, 1972.

Nodine, C. F., \& Lang, N. J. Development of visual scanning strategies for differentiating words. Developmental Psychology, 1971, 5, 221-232.

Nodine, C. F., \& Simmons, F. Development of cognitive strategies for processing distinctive features of letter-like stimuli. Paper presented at the Eastern Psychological Association, Boston, April 1972.

Nodine, C. F., \& Steuerle, N. J. Development of perceptual and cognitive strategies for differentiating graphemes. Journal of Experimental Psychology, in press.

Noton, D., \& Stark, L. Eye movements and visual perception. Scientific American, 1971, 224, 35-43.

Simon, H. A. Complexity and the representation of patterned sequences of symbols. Psychological Review, 1972, 79, 369-382.

Thomas, E. L. Eye movements and fixations during the initial viewing of Rorschach cards. Journal of Projective Techniques \& Personality Assessment, 1963, 27, 345-353.

Vitz, P. C., \& Todd, T. C. A model of the perception of simple geometric figures. Psychological Review, 1971, 78, 207-228.

Zusne, L., \& Michels, M. More on the geometricity of visual form. Perceptual \& Motor Skills, 1962, 15, 55-58.

(Received for publication August 14, 1972; revision received January $19,1973$. 